\title{
GENERALIZATION OF CERTAIN THEOREMS OF G. SZEGÖ ON THE LOCATION OF ZEROS OF POLYNOMIALS
}

\author{
GYULA DE SZ. NAGY
}

Using the theorem of Grace, the following result was obtained by G. Szegö: ${ }^{1}$

Let the polynomial

$$
f(z)=z^{n}+A_{1} z^{n-1}+\cdots+A_{n}
$$

have no zeros in the circular region $|z| \leqq R$. Then the "section"

$$
h(z)=f(z)-z^{n}=A_{1} z^{n-1}+A_{2} z^{n-2}+\cdots+A_{n}
$$

has no zeros in the circular region $|z| \leqq R / 2$.

In case of an even $n$ the example $f(z)=(z-R)^{n}$ shows that the circle $|z| \leqq R / 2$ can not be replaced by a larger concentric circle. But in case $n$ is odd, according to Szegö the polynomial $h(z)$ is different from zero even in the circle $|z| \leqq(R / 2) \sec (\pi / 2 n) .^{2}$

This theorem can be generalized as follows:

I. Let the polynomial

$$
f(z)=z^{n}+A_{1} z^{n-1}+\cdots+A_{n}
$$

have no zeros in the circular region $|z-\alpha| \leqq R$. Then no polynomial

$$
h(z)=f(z),-\epsilon(z-\alpha)^{n}, \quad|\epsilon| \leqq 1,
$$

can have any zeros in the circle $|z-\alpha| \leqq R / 2$.

The example $f(z)=(z-R)^{n}, \alpha=0, \epsilon=1$, shows that this theorem can not be refined even in the case of an odd $n$.

Theorem $\mathrm{I}$ is a consequence of the following more general theorem:

II. Let the polynomial

$$
f(z)=\left(z-a_{1}\right)\left(z-a_{2}\right) \cdots\left(z-a_{n}\right)
$$

have no zeros in the circle $|z-\alpha| \leqq R$; and let the polynomial

$$
g(z)=\left(z-b_{1}\right)\left(z-b_{2}\right) \cdots\left(z-b_{n}\right)
$$

Received by the editors March 27, 1947.

${ }^{1}$ G. Szegö, Bemerkungen zu einem Satz von J. H. Grace über die Wurzeln algebraischer Gleichungen, Math. Zeit. vol. 13 (1922) pp. 28-55.

${ }^{2}$ Loc. cit. p. 46. 
have all its zeros in the circle $|z-\alpha| \leqq \rho, \rho<R$. Then the polynomial

$$
h(z)=f(z)-\lambda g(z), \quad|\lambda| \leqq t^{n}, 0 \leqq t<R / \rho,
$$

can have no zero in the circle

$$
|z-\alpha| \leqq r=\frac{R-\rho t}{1+t} .
$$

In order to prove this we observe first that for any zero $\xi$ of the polynomial $h(z)$

$$
\frac{f(\xi)}{g(\xi)}=\prod_{k=1}^{n} \frac{\xi-a_{k}}{\xi-b_{k}}=\lambda .
$$

Hence $h\left(z_{0}\right) \neq 0$ in every point $z_{0}$ where

$$
\left|\frac{f\left(z_{0}\right)}{g\left(z_{0}\right)}\right|=\prod_{k=1}^{n}\left|\frac{z_{0}-a_{k}}{z_{0}-b_{k}}\right| \neq|\lambda| \text {. }
$$

Now at every point $z_{0}$ of the circular region (4)

$$
\begin{aligned}
\left|z_{0}-a_{k}\right| & \geqq\left|a_{k}-\alpha\right|-\left|z_{0}-\alpha\right| \geqq\left|a_{k}-\alpha\right|-r>R-r \\
& =(r+\rho) t \\
\left|z_{0}-b_{k}\right| & \leqq\left|z_{0}-\alpha\right|+\left|b_{k}-\alpha\right| \leqq r+\rho,
\end{aligned}
$$

so that

$$
\left|\frac{f\left(z_{0}\right)}{g\left(z_{0}\right)}\right|=\prod_{k=1}^{n}\left|\frac{z_{0}-a_{k}}{z_{0}-b_{k}}\right|>\left(\frac{R-r}{r+\rho}\right)^{n}=t^{n} \geqq|\lambda| .
$$

This concludes the proof of II. In the case $b_{1}=b_{2}=\cdots=b_{n}=\alpha$, $|\lambda|=|\epsilon|=1 \quad(\rho=0, t=1)$, we obtain I.

Substituting

$$
g(z)=z^{n}+A_{k} z^{n-k}=z^{n-k}\left(z^{k}+A_{k}\right), \quad \alpha=0, \quad \rho=\left|A_{k}\right|^{1 / k}, \quad \lambda=1
$$

in Theorem II we obtain:

\section{The polynomial}

$$
f(z)=z^{n}+A_{k} z^{n-k}+A_{k+1} z^{n-k-1}+\cdots+A_{n}
$$

has at least one zero in the circle $|z| \leqq 2 r+\left|A_{k}\right|^{1 / k}$ provided the section

$$
h(z)=f(z)-z^{n}-A_{k} z^{n-k} \equiv A_{k+1} z^{n-k-1}+A_{k+2} z^{n-k-2}+\cdots+A_{n}
$$

has at least one zero on the circle $|z| \leqq r$.

The proof of the following theorem is similar to that of II: 
IV. Let $a_{1}, a_{2}, \cdots, a_{n}$ be given points of the complex plane which are all different from $\alpha$. Let $S_{k}$ be the half-plane containing $\alpha$ and having as boundary the perpendicular bisector of $\alpha_{1} a_{k}$. Finally let $S^{*}$ be the common part of the half-planes: $S_{1}, S_{2}, \cdots, S_{n}$. Then no polynomial

$$
h(z) \equiv\left(z-a_{1}\right)\left(z-a_{2}\right) \cdots\left(z-a_{n}\right)-\epsilon(z-\alpha)^{n}, \quad|\epsilon| \leqq 1,
$$

can have a zero in the interior of the convex domain $S^{*}$.

Indeed in any point $z_{0}$ in the interior of $S^{*}$ we have $\left|z_{0}-a_{k}\right|$ $>\left|z_{0}-\alpha\right|$ so that

$$
\left|\frac{f\left(z_{0}\right)}{g\left(z_{0}\right)}\right|=\prod_{k=1}^{n}\left|\frac{z_{0}-a_{k}}{z_{0}-\alpha_{k}}\right|>1 \geqq|\epsilon| .
$$

A theorem similar to II holds also if the zeros of the polynomials $f(z)$ and $g(z)$ are in arbitrary circular domains without common points. One of these circular domains is the interior of a circle, the other the exterior or interior of a circle or a half-plane. Corresponding to these cases three theorems can be obtained generalizing also certain theorems of G. Szegö. ${ }^{3}$

V. Let the zeros of the polynomials $f(z)=\left(z-a_{1}\right) \cdots\left(z-a_{n}\right)$ and $g(z)=\left(z-b_{1}\right)\left(z-b_{2}\right) \cdots\left(z-b_{n}\right)$ be located in the circular regions

$$
|z-\alpha| \geqq \rho_{1} \text { and }|z-\beta| \leqq \rho_{2},
$$

respectively. We assume that these regions have no points in common, that is,

$$
\rho_{1}-\rho_{2}>0, \quad|\beta-\alpha|<\rho_{1}-\rho_{2} .
$$

Then no polynomial

$$
h(z)=f(z)-\epsilon g(z), \quad|\epsilon| \leqq 1,
$$

can have a zero in the interior of the ellipse $E$ with foci at $\alpha$ and $\beta$ and with the major axis $\rho_{1}-\rho_{2}$.

VI. Let the zeros of the polynomials $f(z)$ and $g(z)$ be located in the circular regions

$$
|z-\alpha| \leqq \rho_{1} \text { and }|z-\beta| \leqq \rho_{2},
$$

respectively, such that these regions have no points in common, that is,

$$
|\beta-\alpha|>\rho_{1}+\rho_{2} \text {. }
$$

\section{Then no polynomial}

\footnotetext{
${ }^{3}$ Loc. cit. pp. 47-48, Theorems 13-15.
} 


$$
h(z)=f(z)-\epsilon g(z),
$$$$
|\epsilon|=1 \text {, }
$$

can have a zero in the interior of the hyperbola $H$ with foci at $\alpha$ and $\beta$ and with the real axis $\rho_{1}+\rho_{2}$.

VII. Let the zeros of the polynomials $f(z)$ and $g(z)$ be located in the circular region $|z-\alpha| \leqq \rho$ and in the half-plane $S$, respectively, such that these regions have no points in common. Let $K$ be a conic section with $\alpha$ as focus and the boundary line $L$ of the half-plane $S$ as the directrix corresponding to $\alpha .{ }^{4}$ Then no polynomial

$$
f(z)-\lambda g(z)
$$

with

$$
|\lambda| \geqq t^{n}=\left(e+\rho \frac{e+1}{\delta}\right)^{n}
$$

can have a zero in the interior of the conic section $K$ where $e$ is the numerical eccentricity of $K$ and $\delta$ is the distance of $\alpha$ from the line $L$.

By the interior of a conic section we mean the set of points from which no tangent can be drawn to the given conic section.

In order to prove $\mathrm{V}$ and VI, we denote by $z_{0}$ an arbitrary point in the interior of the conic sections $E$ and $H$, respectively; let

$$
\left|z_{0}-\alpha\right|=r_{1}, \quad\left|z_{0}-\beta\right|=r_{2} .
$$

As to the ellipse $E$ we have

$$
\begin{gathered}
r_{1}+r_{2}<\rho_{1}-\rho_{2} \text { or } \rho_{2}+r_{2}<\rho_{1}-r_{1}, \\
\left|z_{0}-a_{k}\right| \geqq\left|a_{k}-\alpha\right|-\left|z_{0}-\alpha\right|=\left|a_{k}-\alpha\right|-r_{1}>\rho_{1}-r_{1}, \\
\left|z_{0}-b_{k}\right| \leqq\left|b_{k}-\beta\right|+\left|z_{0}-\beta\right| \leqq \rho_{2}+r_{2} .
\end{gathered}
$$

From this Theorem $\mathrm{V}$ follows since

$$
\left|\frac{z_{0}-a_{k}}{z_{0}-b_{k}}\right|>\frac{\rho_{1}-r_{1}}{\rho_{2}+r_{2}}>1 \text { so that }\left|\frac{f\left(z_{0}\right)}{g\left(z_{0}\right)}\right|>1 \geqq|\epsilon| .
$$

As to the hyperbola $H$, we distinguish two cases regarding the position of $z_{0}$, according as $z_{0}$ is nearer to $\beta$ than to $\alpha$, or conversely. In the first case

$$
\begin{gathered}
r_{1}>r_{2}, \quad r_{1} \perp r_{2}>\rho_{1}+\rho_{2}, \text { hence } r_{1}-\rho_{1}>r_{2}+\rho_{2}, \\
\left|z_{0}-a_{k}\right| \geqq\left|z_{0}-\alpha\right|-\left|a_{k}-\alpha\right| \geqq r_{1}-\rho_{1}, \\
\left|z_{0}-b_{k}\right| \leqq\left|z_{0}-\beta\right|+\left|b_{k}-\beta\right| \leqq r_{2}+\rho_{2} .
\end{gathered}
$$

4 That is, the polar of $\alpha$. 
In the second case

$$
\begin{gathered}
r_{1}<r_{2}, \quad r_{2}-r_{1}>\rho_{1}+\rho_{2}, \text { hence } r_{2}-\rho_{2}>r_{1}+\rho_{1}, \\
\left|z_{0}-a_{k}\right| \leqq\left|z_{0}-\alpha\right|+\left|a_{k}-\alpha\right| \leqq r_{1}+\rho_{1}, \\
\left|z_{0}-b_{k}\right| \geqq\left|z_{0}-\beta\right|-\left|b_{k}-\beta\right| \geqq r_{2}+\rho_{2} .
\end{gathered}
$$

Thus we have in the first and second case,

$$
\left|\frac{z_{0}-a_{k}}{z_{0}-b_{k}}\right| \geqq \frac{r_{1}-\rho_{1}}{r_{2}+\rho_{2}}>1, \quad \text { and } \quad\left|\frac{z_{0}-a_{k}}{z_{0}-b_{k}}\right| \leqq \frac{r_{1}+\rho_{1}}{r_{2}-\rho_{2}}<1 \text {, }
$$

respectively. This furnishes Theorem VI since we have

$$
\left|\frac{f\left(z_{0}\right)}{g\left(z_{0}\right)}\right|>1=|\epsilon|, \text { and } \quad\left|\frac{f\left(z_{0}\right)}{g\left(z_{0}\right)}\right|<1=|\epsilon|
$$

respectively.

This proof furnishes the following corollary:

$\mathrm{VI}^{\prime}$. Let $H$ be the hyperbola defined in Theorem VI. No polynomial $f(z)-\lambda g(z)$ with $|\lambda| \geqq 1(|\lambda| \leqq 1)$ can have any zero inside the branch of $H$ containing the focus $\alpha(\beta)$.

Let $z_{0}$ be a point in the interior of the conic section $K$ defined in Theorem VII; let $r$ and $d$ be the distance of $z_{0}$ from the focus $\alpha$ and the directrix $L$, respectively. Then

$$
\frac{r}{d}<e \text { and } \frac{1}{d}<\frac{1}{d^{*}}=\frac{e+1}{\delta} ;
$$

indeed if $z^{*}$ is the point of $K$ nearest to $L$ and $d^{*}$ is the distance of $z^{*}$ from $L$, we have

$$
\frac{\left|z^{*}-\alpha\right|}{d^{*}}=\frac{\delta-d^{*}}{d^{*}}=e .
$$

But $\left|z_{0}-a_{k}\right| \leqq\left|z_{0}-\alpha\right|+\left|a_{k}-\alpha\right| \leqq r+\rho$ and $\left|z_{0}-b_{k}\right| \geqq d$, so that

$$
\begin{aligned}
\left|\frac{z_{0}-a_{k}}{z_{0}-b_{k}}\right| & \leqq \frac{r+\rho}{d}<e+\frac{\rho}{d^{*}}=e+\rho \frac{e+1}{\delta}=t, \\
\left|\frac{f\left(z_{0}\right)}{g\left(z_{0}\right)}\right| & \leqq\left(\frac{r+\rho}{d}\right)^{n}<t^{n}=|\lambda| .
\end{aligned}
$$

This establishes the proof of Theorem VII.

The special cases 


$$
b_{1}=b_{2}=\cdots=b_{n}=0, \beta=0, \rho_{2}=0, \epsilon=1 \quad \text { of } \mathrm{V} \text { and VI, }
$$

and

$$
a_{1}=a_{2}=\cdots=a_{n}=0, \alpha=0, \rho=0, e=1, \lambda=1 \quad \text { of VII, }
$$

are due to G. Szegö.

UNIVERSITY OF HUNGARY, SZEGED

\section{SOME REMARKS ON POLYNOMIALS}

\section{P. ERDÖS}

This note contains some disconnected remarks on polynomials.

Let $f_{n}(x)=\prod_{i=1}^{n}\left(x-x_{i}\right), \quad-1 \leqq x_{1} \leqq x_{2} \leqq \cdots \leqq x_{n} \leqq 1$. Denote by $-1 \leqq y_{1} \leqq \cdots \leqq y_{n-1} \leqq 1$ the roots of $f_{n}^{\prime}(x)$. We prove the following theorem.

THEOREM 1. For all $n$

$$
\left|f_{n}(-1)\right|+\left|f_{n}(+1)\right|+\sum_{i=1}^{n-1}\left|f_{n}\left(y_{i}\right)\right| \leqq 2^{n} \text {. }
$$

For $n \geqq 3$

$$
\left|f_{n}(-1)\right|^{1 / 2}+\left|f_{n}(+1)\right|^{1 / 2}+\sum_{i=1}^{n-1}\left|f_{n}\left(y_{i}\right)\right|^{1 / 2} \leqq 2^{n / 2} .
$$

For $n \geqq n_{0}(k)$

$$
\left|f_{n}(-1)\right|^{1 / k}+\left|f_{n}(+1)\right|^{1 / k}+\sum_{i=1}^{n-1}\left|f_{n}\left(y_{i}\right)\right|^{1 / k} \leqq 2^{n / k} .
$$

REMARK. If $y_{i}=y_{i+1}$ or $-1=y_{1},+1=y_{n-1}$ the corresponding summands clearly vanish.

Clearly

$$
\begin{gathered}
\left|f_{n}(-1)\right| \leqq \\
\left(1-x_{1}\right) 2^{n-1}, \quad\left|f_{n}\left(y_{i}\right)\right| \leqq\left|y_{i}-x_{i+1}\right| 2^{n-1}, \\
\left|f_{n}(+1)\right| \leqq\left(1-x_{n}\right) 2^{n-1} .
\end{gathered}
$$

Thus

Received by the editors March 28, 1947. 